\title{
Localized Secure Examination Printing Service with Remote Locking
}

\author{
Harshitha D, Kavya R, Lavanya M, Komal G, Gururaj KS* \\ Department of Computer Science and Engineering, GSSS Institute of Engineering and \\ Technology for Women, Mysuru, India
}

DOI: https://doi.org/10.21467/proceedings.1.9

* Corresponding author email: gururaj.ks@gsss.edu.in

\begin{abstract}
Examination is conducted to test a person's knowledge or proficiency in a subject. An examination can be a written test or can be conducted online. The major drawback of written test is less security of the system. Question paper may get leaked, this is one of the security issue with written test system. This may result in reexamination or may lower the interest of student. Remote locking system can be used to overcome this problem, where question paper gets printed without human intervention and will not be visible to anyone until the lock is released.
\end{abstract}

Index Terms- Paper locking system, Question paper leakage, Remote locking, Without human intervention.

\section{INTRODUCTION}

Every exam postponed is every active mind being set back in terms of performance. It is a common issue that examination papers are being leaked one or the other during transit or internal leaks. Due to high availabilities and cheaper cost of local printing at current date, it is much observable that one of the common leak factor i.e. via transit can be mitigated by printing the papers locally with geographical Identities and tracking the internal transit till the examination hall. The system stores the data in encrypted format in the cloud server until the main admin releases the codes to the sub nodes available. Every node will be active only when the admin explicitly releases the lock via cloud server, where in, every node will be actively listening to the server by keeping an TCP connection alive by using protocol such as MQTT developed by IBM and being an OASIS standard for textual communication.

Examination paper leak has become common in many sectors of education. This is not only hampers the culture of exam but also discourage students. Student's active mind is set back due to exam being postponed. Leak of the examination materials can take place at any level. It may be from where it is shipped, treasury officer officials, from center where these papers are stored [4]. This can be mitigated by printing the papers locally with geographical identities and also by tracking internal transit. Every exam postponed is every active mind being set back in terms of performance.

(C) 2018 Copyright held by the author(s). Published by AIJR Publisher in Proceedings of the $3^{\text {rd }}$ National Conference on Image Processing, Computing, Communication, Networking and Data Analytics (NCICCNDA 2018), April 28, 2018.

This is an open access article under Creative Commons Attribution-NonCommercial 4.0 International (CC BY-NC 4.0)

license, which permits any non-commercial use, distribution, adaptation, and reproduction in any medium, as long as the

AijR license, which permits any non-commercial use, distribution, 


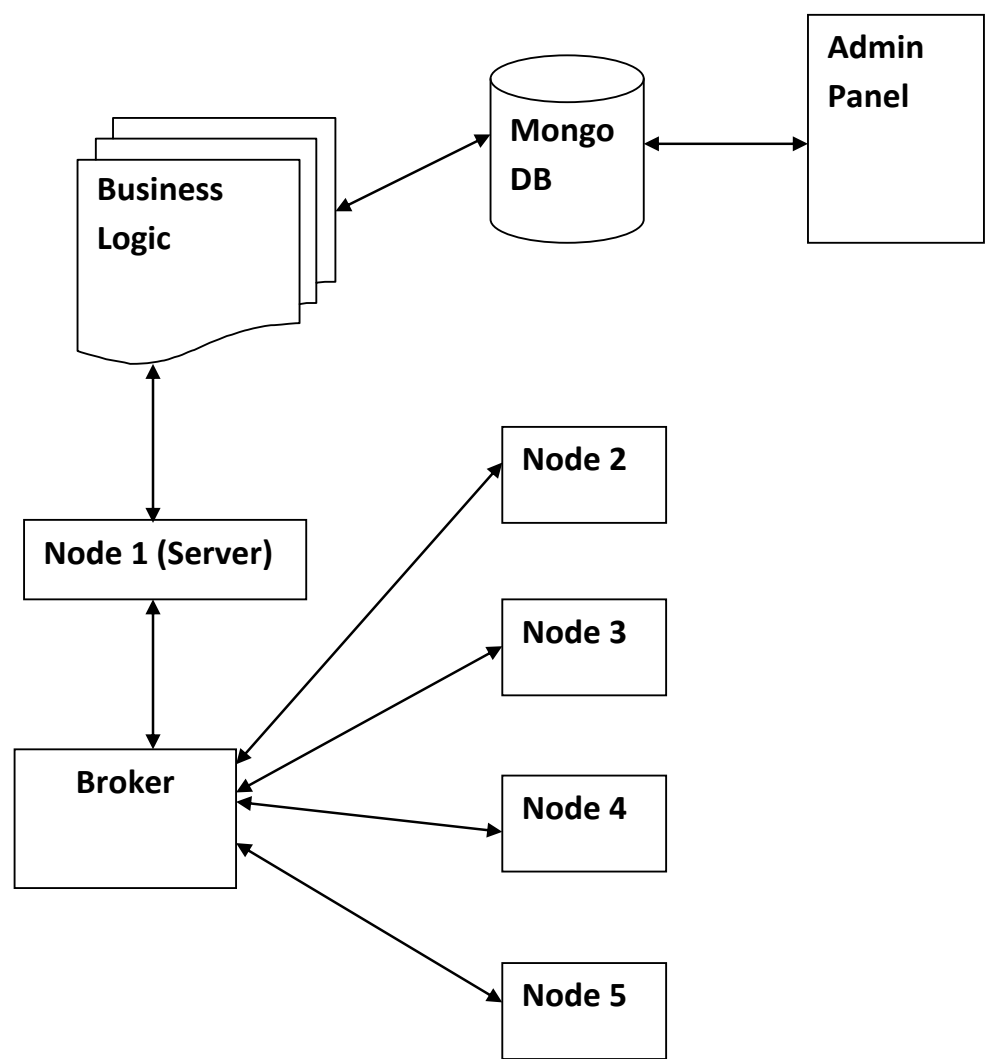

Fig1: Architecture of the project.

\section{LITERATURE SURVEY}

Literature survey is an important step in software development process. In various referred papers, authors have discussed about different factors that affect security of examination process, methods used to enhance examination security, techniques used in online exams. The papers suggest that various protocols, techniques that can be used to overcome drawbacks of the existing examination system.

\section{Question paper delivery system}

There are various methods for delivering the question paper. One method may be printing in one center and transporting it to all institutions. Another method is through online delivery of questions papers [1] [11]. In some cases there are software used to set question paper[2].Even online exam question paper delivery system may lead to serious lapses during examination . To overcome this password protected question paper delivery is done. There are some companies who takes the responsibility of delivering question paper online[8]. Examination is a complex process and confidentiality is very important automated management information

Proceedings of the $3^{\text {rd }}$ National Conference on Image Processing, Computing, Communication, Networking and Data Analytics (NCICCNDA 2018) 
Localized Secure Examination Printing Service with Remote Locking

system which is controlled by workflow and security is ensured through dynamic database authentication [9].

\subsection{MQTT protocol}

Message queuing telemetric transport is publishing subscribe based messaging protocol. TCP and UDP protocols are used for IOT appliances. There are various other protocols XMPP, DDS, AMQP used to handle various issues. It represents M2M protocol. MQTT protocol has several benefits like increased scalability, reduction in bandwidth consumption, efficient distribution of information. This protocol connects devices and network with middleware and applications. The default port where it works is on TCP/IP port. The various types of MQTT arehivemq, pahoMQTT. It is most widely used connection protocol for M2M and IOT [3][10].

\subsection{Question paper leaks}

In the usual procedure of examination, the major issue is question paper leakage. When question papers are leaked, re-examination may be done again [6]. There are many levels where question papers can be leaked. The faculty who sets the question paper may leakit, or staff who is handling the computer system where question papers are stored might leak it [4]. The leak paper reaches students through e-mail, sms orwhatsaap group [5]. There are several fake leak mafias who make money from this process [7]. Even if the higher authority gets to know about the leak at the last minute proper actions cannot be taken.

\section{REQUIRMENT ANALYSIS}

To achieve the aims of this system, the system requirements need to be determined. In this part the detail of the requirements will be determined in the following steps:

\subsection{System Requirements}

Functional Requirements: these requirements are waysused to determine the functions of the system or thecomponents of these functions. It could be processing data, calculations, organizing instructions or other things needed to be done by the system regarding user requirements.

The functional requirements of our project are:

Question paper upload: Allows faculties to upload question paper files.

File conversion: It converts those files to pdf files.

Unique Identifier: System adds unique identifier to each paper and it will be generated by random number generator.

The software requirements of this project are:

1. Database: MongoDB

2. Backend Language: PHP (Server-Python Flask)

Python(Client/Nodes)

PHP(Broker-Aedes).

3. Frontend Language: HTML, CSS, JS. 
Hard ware requirements are:

1. System processor: $1.8 \mathrm{GHz}$ or more.

2. System RAM: $1 G B$ or more.

3. Processor bit: 32bit or 64bit.

4. Hard disk space: $20 \mathrm{~GB}$ or more.

\section{DESIGN}

The two major components of system development are system analysis and system design. The system architecture is established in system design phase. It maps the requirement into architecture. The components, their interfaces and behaviors are defined in architecture. The design document describes a plan to implement the requirement.

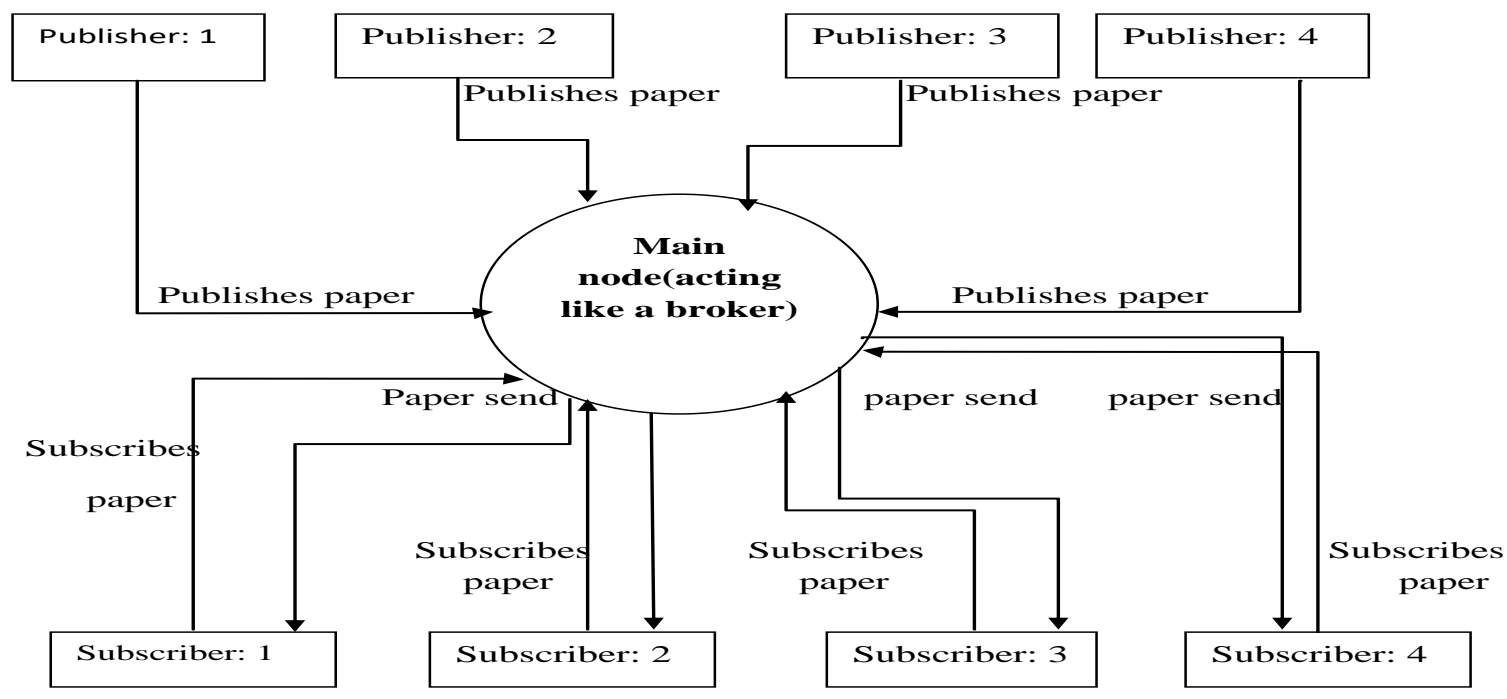

Fig2: Data Flow diagram showing communication between publisher and subscriber.

Fig2 depicts the high-level design, where the broker acts as the mediator between the publisher and the subscribers. Faculties from different institutions will upload the examination question papers from various locations to the server directly. Once all the authentication process got verified server only select question papers by using random number generation method and sends to the broker later broker distributes to all the subscribers.

Proceedings of the $3^{\text {rd }}$ National Conference on Image Processing, Computing, Communication, Networking and Data Analytics (NCICCNDA 2018) 


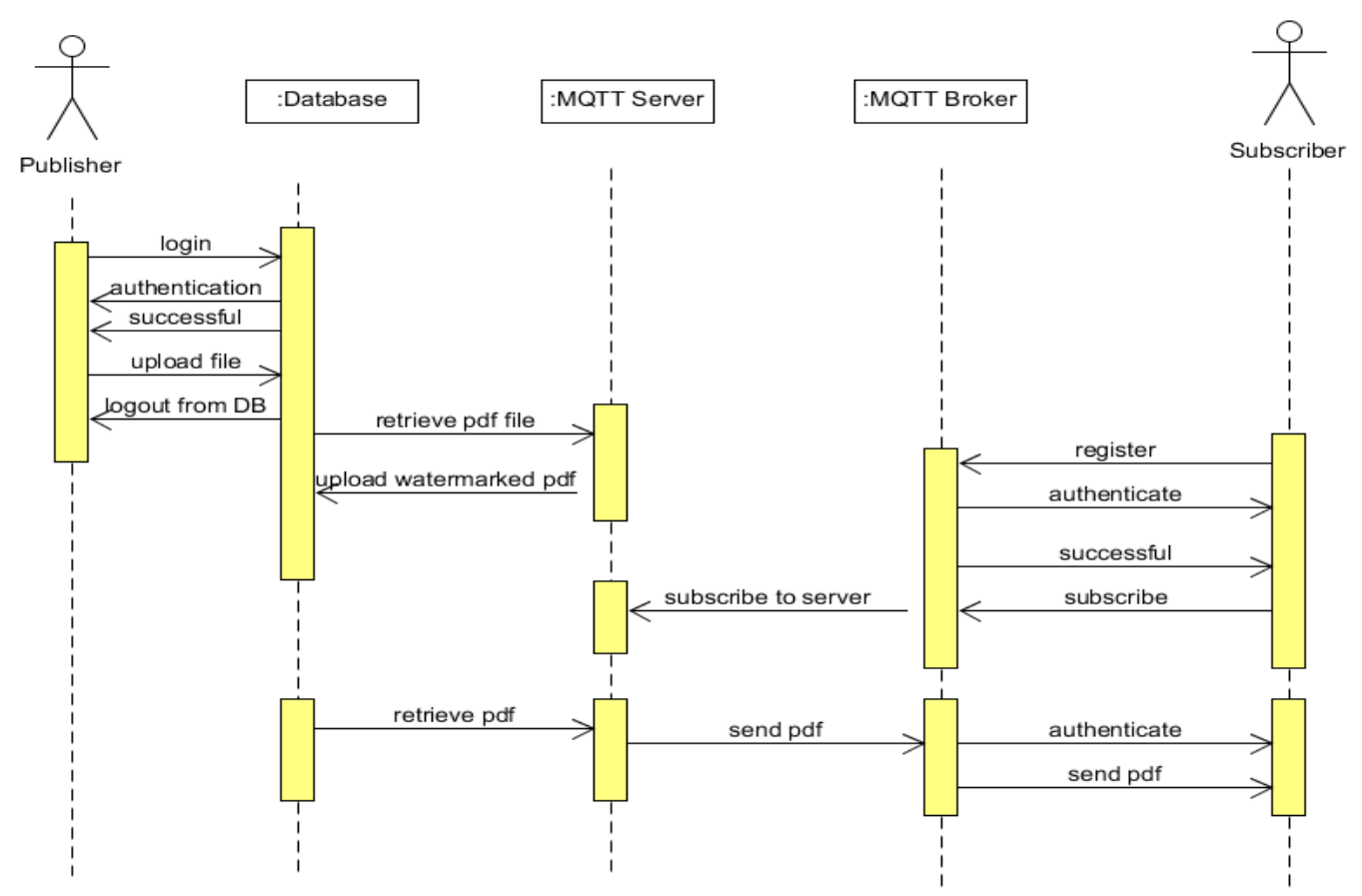

Fig3: Sequence diagram representing events.

Fig3 shows the Sequence diagram of the system. Initially, both the publisher and the subscribers register themselves with the server in cloud system. The publishers need to register with username, password, college details. Later they need to use same credentials to login and upload the paper.

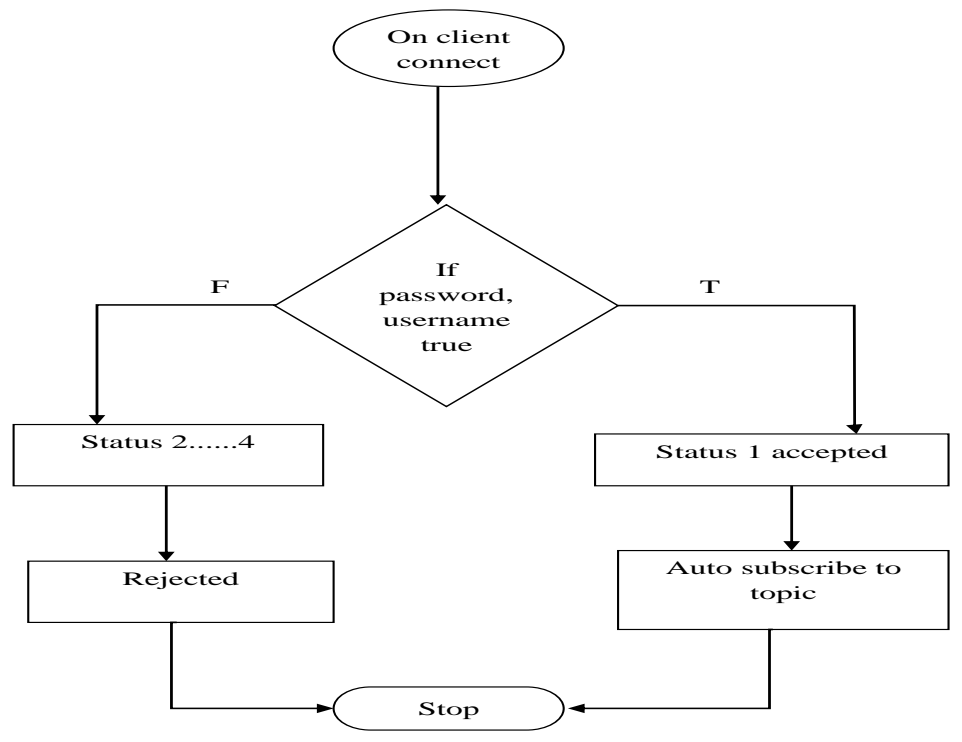

Fig4: Flow diagram for authentication. 
Fig 4 describes that if the password and username (authentication elements) once verified and validated as true then client is accepted and auto subscribe to the topic otherwise the client is treated as unknown person and rejected.

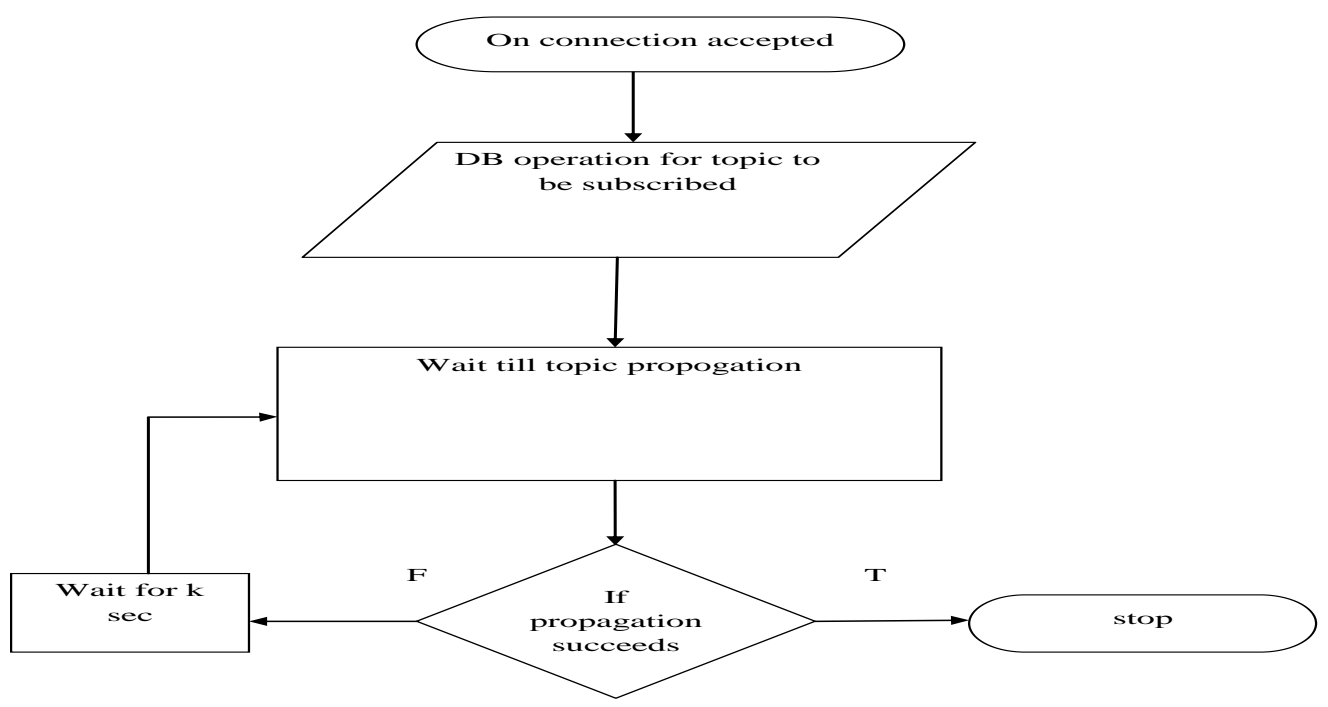

Fig5: Flow diagram for topic authority controller.

Fig5 shows that once the publisher side connection is accepted database operation for topic is subscribed and further if the propagation succeeds the process will terminates otherwise it will wait for $\mathrm{k}$ seconds and later when the propagation succeeds it terminates.

\section{CONCLUSION}

Examination decides one's skill and proficiency in a subject. When it is conducted online it has more security feature. The process of written test is tedious, consumes more time and chances of paper being leaked is more. This is caused by human interaction with the system where the paper is stored, if human intervention is removed paper leaked can be avoided. So, when question papers are directly printed in institutions on the examination day, without human intervention with the system security of examination can be enhanced. As of now design of the system is completed. Further work will be done to implement the remotelocking service for examination.

\section{References}

[1] THE HINDU "VTU will experiment with online delivery of question papers this year" (online), published: 10-042012, Accessed on: 18-04-2018

[2] "Computers to set board exam papers, a major technical reform", http://digitallearning.eletsonline.com, published: 27-03-2017, accessed on: 18-04-2018

[3] Muneer Bani Yassein, Mohammed Q. Shatnawi, ShadiAljwarneh, Razan Al-Hatmi, "Internet of Things: Survey and open issues of MQTT Protocol”, pp.57-62, 2017.

Proceedings of the $3^{\text {rd }}$ National Conference on Image Processing, Computing, Communication, Networking and Data Analytics (NCICCNDA 2018) 
Localized Secure Examination Printing Service with Remote Locking

[4] BANGALORE MIRROR, "Source of VTU exam paper leak in city engineering college" (online), published :1704-2018, accessed on: 18-04-2018

[5] "VTU question paper leaked through e-mail, sms" http://www.dnaindia.com, published on: 17-01-2013, accessed on: $18-04-2018$

[6] "CBSE re-exam for class 10 maths only in Delhi,Haryana", www.business-standard.com, published :31-03-2018, accessed on: 18-04-2018

[7] THE INIAN EXPRESS, "HRD ministry warns students against fake leak mafias" (online), published : 17-04-2018, accessed on: 18-04-2018

[8] "Mindlogicx", https://www.mindlogicx.com.

[9] Hnixu, Rongxiong Guo, LitingLei,Rongrong Fan, "Deign and implementations of examination affairs management system”, $20163^{\text {rd }}$ international conference on Information Science and control emerging, pp.294-296, 2016.

[10] Syed Naeem Firdous,ZubairBaig,Craigvalli, Almed Ibrahim, "Modeling and evaluation of malicious attacks against the IOT ,MQQT protocol, IEEE International conference on Internet of Things and IEEE green computing and communication pp.748-755, 2017.

[11] Guang Cena, Yuxiao Donga, Wanlin Gao, Lina Yua, Simon See, Qing Wang, Ying Yang, Hongbiao Jiang, “A implementation of an automatic examination paper generation system" https://www.journals.elsevier.com, 2010. 